\title{
Evaluation einer zweijährigen Intensivbetreuung von jungen Menschen mit Schizophrenie
}

\section{Evaluation of a two-year intensive outpatient care programme for adolescents with schizophrenia}

\author{
M. J. Hemmerle ${ }^{1}$, B. Röpcke ${ }^{2}$, C. Eggers ${ }^{2}$ und R.D. Oades ${ }^{2}$
}

1 Prof. Eggers-Stiftung, Essen, Germany,

2 Klinik für Psychiatrie und Psychotherapie des Kindes- und Jugendalters, LVR-Kliniken

Essen, Universität Duisburg-Essen, Germany.

(2010) Zeitschrift für Kinder- und Jugendpsychiatrie und Psychotherapie, 38 (5), 361-369 DOI 10.1024/1422-4917/a000060

"This article does not exactly replicate the final version published in the Zeitschrift für Kinder- und Jugendpsychiatrie und Psychotherapie. It is not a copy of the original published article \& is not suitable for citation."

Zusammenfassung. Fragestellung: Im Projekt «Trialog» werden Jugendliche, die an Schizophrenie mit frühem Beginn (EOS) leiden, zwei Jahre im Anschluss an die stationäre Behandlung intensiv betreut, um Recovery und Autonomie zu fördern. Die multiprofessionelle Betreuung umfasst psychoedukative (Multi-)Familienarbeit, Bewältigung persistierender positiver Symptome, Aufbau sozio-emotionaler Kompetenz, Alltags- und Freizeittraining sowie Unterstützung bei der Schul- und Berufsausbildung. Methodik: Psychopathologie, soziales und neuropsychologisches Funktionsniveau von zwölf Bewohnern wurden über zwei Jahre verfolgt. Ihr Fortschritt wurde mit zwölf EOS-Patienten verglichen, die nach der Entlassung nicht im Projekt «Trialog» betreut wurden. Ergebnisse: Teilnehmer zeigten einen Rückgang positiver und negativer Symptome, während positive Symptome in der Vergleichsgruppe anstiegen. Das soziale Funktionsniveau stieg (GAF), ein höherer Anteil von Betreuten lebte im Anschluss selbstständig und ging einer regelmäßigen Tätigkeit nach. Neuropsychologische Indikatoren des Gedächtnis (WMS-R), der (selektiven) Aufmerksamkeit (TMT), der visuo-motorischen Koordination (MT) und des psychomotorischen Tempos (ZS) besserten sich stärker als in der Vergleichsgruppe. Keine Gruppe zeigte Veränderungen in Maßen der Intelligenz oder der subjektiven Lebensqualität (MANSA). Schlussfolgerung: Erste Analysen geben Hoffnung, dass mit dem Projekt «Trialog» die Betreuung für EOS-Patienten in der kritischen Phase in den ersten Jahren nach Beginn der Schizophrenie innerhalb der Regelversorgung verbessert werden kann.

Schlüsselwörter: Schizophrenie mit frühem Beginn, Wirksamkeit, Intensivbetreuung, soziales Funktionsniveau, neuropsychologisches Funktionsniveau

\begin{abstract}
.
Objective: The Trialog project offers patients with early-onset schizophrenia (EOS) a two-year programme of residential outpatient care following discharge from a clinic. The programme aims to support their further recovery and independence and encompasses interactive and psychoeducational multifamily care, coping with persistent symptoms, development of socio-emotional competence, independent house keeping, and support of school and vocational training. Methods: To evaluate psychopathology along with social and neuropsychological function for 12 participants over a period of 2 years. Their progress was compared with that of 12 EOS patients who did not attend Trialog following discharge. Results: Participants showed a significantly greater decrease of positive and negative symptoms, as opposed to an increase in positive symptoms in the comparison group. Measures of social function, neuropsychological indicators of memory, (selective) attention, and psychomotor speed improved more than in the comparison subjects. Neither group showed changes in measures of intelligence or in the subjective quality of life. Conclusions: First analyses raise hope that monitoring participant performance in the programme of the "Trialog project" will improve the treatment and care of EOS-patients in the critical first years following the onset of schizophrenia.
\end{abstract}

Keywords: clinical outcome, early-onset schizophrenia, intensive care, social function, neuropsychology 


\section{Einleitung:}

Schizophrenie führt zu einer funktionellen Degeneration von Teilen des Gehirns infolge einer gestörten Entwicklung des Nervensystems (Falkai, 2008; White, Ward, O'Leary \& Andreasen, 2006). Dies kann zu anhaltenden Beeinträchtigungen führen (Röpcke \& Eggers, 2005), die eine umfassende Betreuung nach der Entlassung aus der Klinik erforderlich machen, vor allem bei frühem Beginn der Störung (Early Onset Schizophrenia, EOS, Biswas, Malhotral, Malhotral \& Nitin, 2006a). Es ist für viele Betroffene schwer, einen Schulabschluss oder eine Berufsausbildung zu erwerben (Eggers, Bunk, Volberg \& Röpcke, 1999; Röpcke \& Eggers, 2005). Da Schäden an Genen als Ursache von Schizophrenie bislang nicht gefunden wurden und sich auch die Befürchtung einer neurotoxischen Wirkung der Schizophrenie nicht bestätigte (Kircher \& Gauggel, 2008) bleibt die Hoffnung bestehen, entwicklungs- und damit remissionsfördernde Bedingungen vor allem in den kritischen ersten Jahren nach Beginn der Störung weiter verbessern zu können. Die Wirkung intensiver psychotherapeutischer und sozialer Interventionen bei Erwachsenen mit Schizophrenie (Adult Onset Schizophrenia, AOS) ist bekannt (Benedetti 1998, Ciompi, Hoffmann \& Broccard, 2001; Katschnig, Konieczna, Michelbach \& Sint, 1989).

Ein spezialisiertes Angebot zur Rehabilitation betroffener Jugendlicher besteht für den deutschsprachigen Raum im Einzugsbereich der Universitätsklinik Marburg seit 1979 (Martin, 1991). Eine Verlaufstudie über ein Jahr (Martin, 1991) zeigte eine Stabilisierung der psychopathologischen Symptomatik und eine teilweise Besserung neuropsychologischer Defizite der dort Betreuten. In einer Follow-up Studie der Patienten mit EOS der Klinik Marburg nach neun Jahren
(Fleischhaker et al., 2005) fand sich kein Unterschied von dort betreuten Patienten zu denen, die nicht in dieser Einrichtung betreuten wurden. Dies ist ein Erfolg, da dieser stationären Rehabilitation besonders früh und schwer Betroffene zugewiesen wurden. Eine retrospektive Analyse der Symptomatik und der sozialen Situation nach im Mittel acht Jahren (Heinemann, 2005) zeigte allerdings keine dauerhafte Verbesserung des Verlaufes. Viele der Betreuten kamen aus großer Entfernung und erlitten beim Ortswechsel sowohl zu Beginn, als auch zum Abschluss der Betreuung, einen umfassenden Abbruch aller Umweltbezüge. Gleichzeitig ist es den Familien in großer Entfernung kaum möglich, die Entwicklung ihrer Kinder mit zu vollziehen und gemeinsame Strategien mit Therapeuten und Betreuern zu entwickeln.

Für AOS-Patienten wurde diese Situation in Wien mit der Betreuung in einer gemeindenahen, familienzentrierten Wohngruppe verbessert (Katschnig et al., 1989). Eine Untersuchung drei Jahre nach dem Auszug zeigte, dass die dort Betreuten anschließend weniger häufig stationär behandelt wurden. Etwa zwei Drittel gingen einer regelmäßigen Tätigkeit nach und wohnten selbstständig (MayrMauhart, 2005). Die Mitarbeit der Familien schien einer der Hauptfaktoren zu sein, der zu weiteren Fortschritten und einer Stabilisierung des Erreichten beitrug. Um auch EOS-Patienten eine vergleichbare Möglichkeit zu geben wurde das Projekt «Trialog» entwickelt und begann seine Arbeit im Jahre 2002 in Essen.

In der Wohngruppe Trialog (WG Trialog) werden Jugendliche und junge Erwachsene intensiv mit einem Stellenschlüssel von 1:1 von einem multiprofessionellen Team (Sozialarbeiter/ -innen, Erzieherinnen, Krankenpflegerin, Ergotherapeut, Psychologischer Psychotherapeut) 24 Stunden täglich betreut und 
behandelt. Selbstversorgung und Ausbildung werden gefördert. Es besteht enger Kontakt zu einer Schule für Kranke und Anbietern von Arbeitstrainings- und Berufsvorbereitungsmaßnahmen. Anforderungen werden flexibel an die Belastbarkeit angepasst. Durch gemeindenahe, vernetzte Arbeit wird ein gleichzeitiger Wechsel in mehreren Lebensbereichen bei Ein- und Auszug vermieden. Eine intensive, partnerschaftliche Familienarbeit («Trialog») erfolgt in flexiblen Settings (Familien-, Elterngespräche, Multifamiliengruppen mit mehreren Familien (McFarlane, Dushay, Stastny, Deakins \& Link, 1996), Telefonkontakte, gemeinsame Aktivitäten). Wenn erforderlich, erfolgen täglich Telefonate mit den Eltern und wöchentlich Gespräche. Die ganze Familie wird bei der Bewältigung der Schizophrenie unterstützt. Die Betreuung ist auf zwei Jahre begrenzt, um Regression zu vermeiden und erreichbare Ziele für die weitere Lebensführung $\mathrm{zu}$ suchen und $\mathrm{zu}$ verfolgen. Auch die Bewilligung durch Kostenträger (meist Jugendämter) wird durch die Befristung erleichtert. Es besteht ein anschließendes Angebot ambulant betreuten Wohnens beim gleichen Träger, das von $67 \%$ der Bewohner wahrgenommen wird (Tabelle 2).

Gruppen- und Maltherapie (Benedetti, 1998, 2004), Entspannungs- und Stressbewältigungstraining sind neben regelmäßigen und bedarfsorientierten Einzelgesprächen tägliches Angebot. Die vorliegenden Therapiemanuale zur Psychoedukation für Erwachsene bzw. Familien mit schizophrenen Störungen (Übersicht: Hemmerle, 2008) wurden für die Arbeit mit jungen Menschen adaptiert. Ein Schwerpunkt wurde auf die Stabilisierung der Identität und den Abbau von Selbststigmatisierung gelegt (Benedetti, 2004; Eggers, 2004). Einmal wöchentlich visitiert ein Facharzt für Psychiatrie und Psychotherapie des Kindes und
Jugendalters, im Krisenfall erfolgt eine Vorstellung in der Ambulanz der zuständigen Klinik.

Mit dieser Studie soll untersucht werden, ob der Verlauf von EOS sich unter einer solchen Behandlung und Betreuung im Vergleich zu einer Regelbehandlung (Treatment as usual, TAU) verbessert. Dabei interessiert insbesondere, ob sich ein stärkerer Rückgang psychopathologischer Symptome zeigt, bei Betroffenen mit prognostisch ungünstigen Merkmalen (schleichender Krankheitsbeginn, schlechte prämorbide Anpassung (Röpcke \& Eggers, 2005) eine bessere Remission erzielt werden kann, die subjektive Lebensqualität steigt, sich das soziale und neuropsychologische Funktionsniveau in höherem Maße bessert und die untersuchte Form der Betreuung effektiv in eine Jugendhilfeeinrichtung der Regelversorgung implementiert werden kann.

\section{Methodik:}

Im Zeitraum der Datenerhebung für diese Untersuchung (10/2002 bis $12 / 2006)$ zogen 24 Bewohner in die Wohngruppe Trialog ein. Ein junger Mann zog nach einem Monat wieder aus. Eine junge Frau, die an einer Borderline-Störung litt und aus Gründen des Gleichgewichts der Geschlechter aufgenommen wurde, zog nach einem Jahr in eine eigene Wohnung. In zwei Fällen kehrten die Söhne zu ihren Familien zurück. Drei junge Männer wurden disziplinarisch wegen Weitergabe von Drogen in der Wohngruppe bzw. aggressiven Verhaltens entlassen. Eine Bewohnerin lehnte eine Testung ab. Zwei Betreute waren aufgrund unzureichender Kenntnisse der deutschen Sprache bei Migrationshintergrund nicht testbar. Zwei weitere nahmen vor dem Einzug an der Studie teil, aus der sich die Vergleichsgruppe rekrutierte (s. u.), und wurden dort berücksichtigt, um gleiche Teilnehmerzahlen in beiden Gruppen zu erzielen. 
Tab. 1: Demografische and klinische Merkmale der Trialog-Gruppe (TG) und der Vergleichsgruppe (VG): Mittelwerte $b z w$. Anzahl, Standardabweichungen bzw. Prozent in Klammern, Minima und Maxima

\begin{tabular}{|c|c|c|c|}
\hline \multicolumn{2}{|l|}{ Variablen } & TG $(\mathrm{N}=12)$ & $\mathbf{C G}(\mathrm{N}=12)$ \\
\hline \multirow[t]{3}{*}{ Alter (Jahre) } & 1. Aufnahme: & $16.6(1.0) 15.55-17.33$ & $17.2(1.3) 14.72-19.35$ \\
\hline & T1: & $17.5(0.6) 16.63-18.53$ & 17.4 (1.4) $14.73-19.38$ \\
\hline & T2: & $19.4(0.5) \quad 17.96-20.41$ & 20.2 (1.8) $17.95-23.76$ \\
\hline \multirow{2}{*}{$\begin{array}{l}\text { Geschlecht, } \\
\text { Anzahl (\%) }\end{array}$} & Weiblich & $5 \quad(41)$ & $3(25)$ \\
\hline & Männlich & $7 \quad(58)$ & $9 \quad(75)$ \\
\hline \multirow{2}{*}{$\begin{array}{l}\text { Diagnose (ICD-10), Anzahl, } \\
(\%)\end{array}$} & Schizophrenie & $10(83)$ & $12(100)$ \\
\hline & Schizoaffektive & 2 (17) & 0 \\
\hline \multicolumn{2}{|c|}{ Dauer der unbehandelten Psychose ${ }^{1}$ Jahre, } & $1.09(0.6) \quad 0.34-2.33$ & $0.5(0.7) \quad 0.02-.33$ \\
\hline \multirow{2}{*}{$\begin{array}{l}\text { Antipsychotika, } \\
\text { chlorpromazinäquivalente mg } \\
\text { pro Tag, }\end{array}$} & $\mathrm{T} 1$ & 421 (133) 240-666 & 468 (200) $240-900$ \\
\hline & $\mathrm{T} 2$ & $563(258) 200-1000$ & $531(366) 267-1180$ \\
\hline \multirow{6}{*}{$\begin{array}{l}\text { Sozioökonomischer Status }{ }^{2} \text {, } \\
\text { Anzahl (\%) } \\
\text { (I kam nicht vor) }\end{array}$} & II & $1(8)$ & $4(33)$ \\
\hline & III & $3(25)$ & $3(25)$ \\
\hline & IV & $0(0)$ & $2(17)$ \\
\hline & $\mathrm{V}$ & $3(25)$ & $3(25)$ \\
\hline & VI & $3(25)$ & $0(0)$ \\
\hline & VII & $2(17)$ & $0(0)$ \\
\hline
\end{tabular}

1: $\mathrm{F}_{1,22}=4.768 / \mathrm{p}=.041$

2: Kendall tau-c $=.528 ; \gamma=2.806 / \mathrm{p}=.005$

Die verbleibenden 12 Bewohner erfüllten die ICD-10 Kriterien für F20 Schizophrenie oder F25 schizoaffektive Störungen. Dem Einzug in die WG Trialog gingen stationäre Behandlungen in verschiedenen Kliniken für Psychiatrie und Psychotherapie des Kindes- und Jugendalters voraus. Die Betreuung in der WG Trialog wurde von den Kliniken oder den zuständigen Jugendämtern vorgeschlagen. Die erste Untersuchung (T1) der TrialogGruppe (TG) erfolgte zu Beginn der Betreuung, die zweite Untersuchung (T2) nach zwei Jahren bei Abschluss der Betreuung, drei Jahre nach der ersten Klinikaufnahme (Tabelle 1). Alle Teilnehmer stimmten der Teilnahme an der Untersuchung zu. Bei Minderjährigen erfolgte die Zustimmung auch durch die Eltern.

Diese Studie ist die Fortsetzung einer multimodalen Verlaufsstudie von jungen Patienten mit Schizophrenie (Oades et al., 2006; Oknina et al., 2005), deren Übereinstimmung mit der Deklaration von Helsinki vom Ethik-Rat der Universitätskliniken Essen bestätigt wurde. Aus dieser Studie wurde eine Vergleichsgruppe (VG) von 12 EOS-Patienten gewonnen, die im Alter zwischen 14 und 19 Jahren in der Universitätsklinik für Psychiatrie und Psychotherapie des Kindes- und Jugendalters Essen behandelt wurden. Sie und ihre Eltern waren mit Untersuchungen während und drei Jahre nach der stationären Behandlung einverstanden. Im Anschluss an die Behandlung in der Klinik erfolgte ein TAU.

In dieser Studie verwendete Verfahren (s. u.) konnten übernommen werden, da die damit erhobenen Daten geeignet waren, die hier untersuchten Fragestellungen zu beantworten. Alter, Geschlecht, Diagnosen, Medikation (Chlorpromazinäquivalente) und der sozioökonomische Status (Brauns, Haun \& Steinemann, 1997) von beiden Gruppen sind in Tabelle 1 zusammengefasst.

Psychopathologische Symptome wurden mit der Positive and Negative Syndromes 
Scale (PANSS; Kay, 1987) in der TG und den Scales for the Assessment of Negative/Positive Symptoms (SANS/SAPS; Andreasen, 1983, 1984) in der VG erhoben. Die hier betrachteten Summenwerte zeigen gute Übereinstimmung (Norman, Malla, Cortese \& Diaz, 1996). Die Schwere der Erkrankung wurde mit dem Clinical Global Impression (CGI) des National Institute of Mental Health eingeschätzt. Zusätzlich wurden mit der Manchester Short Assessment of Quality of Life (MANSA; Priebe, Huxley, Knight \& Evans, 1999, deutsche Übersetzung von Röpcke und Linau, s. Hemmerle, 2008) die Zufriedenheit mit verschiedenen Lebensbereichen erhoben.

Tab. 2: Wohnbedingungen, regelmäßige Tätigkeiten und Schulbesuch zu T2 für Teilnehmer der TG und der VG $(\%$ in Klammern)

\begin{tabular}{|c|c|c|}
\hline Variable & $\begin{array}{l}\text { TG } \\
(\mathrm{N}=12)\end{array}$ & $\begin{array}{l}\mathrm{VG} \\
(\mathrm{N}=12)\end{array}$ \\
\hline Wohnbedingungen zu $T 2^{1}$ Anzahl (\%) & $N(\%)$ & $N(\%)$ \\
\hline Eigene Wohnung & $1(8)$ & $1(8)$ \\
\hline $\begin{array}{l}\text { Wohngemeinschaft und } \\
\text { ambulant betreutes Wohnen }\end{array}$ & $8(67)$ & $1(8)$ \\
\hline Wohnen bei Eltern & $2(17)$ & $7(58)$ \\
\hline Wohnheim & $1(8)$ & $3(25)$ \\
\hline \multicolumn{3}{|l|}{ Regelmäßige Tätigkeit zи $T 2^{2}$} \\
\hline Berufsausbildung & 0 & $1(8)$ \\
\hline Berufsvorbereitende Maßnahme & $4(25)$ & $1(8)$ \\
\hline Freiwilliges soziales Jahr & $1(8)$ & 0 \\
\hline Schule & $2(17)$ & $3(25)$ \\
\hline Beschützte Arbeit & $2(17)$ & 0 \\
\hline Tagesstätte & $1(8)$ & 0 \\
\hline Summe Tätigkeiten & $10(83)$ & $5(42)$ \\
\hline Keine regelmäßige Tätigkeit & $2(17)$ & $7(58)$ \\
\hline Schulabschluss zu T2 Anzahl (\%) & $6(50)$ & $7(58)$ \\
\hline
\end{tabular}

Es wurden Merkmale der Lebenssituation (Wohnbedingungen, Schulabschluss und aktuelle regelmäßige Aktivitäten zum Zeitpunkt T2) nach steigenden Anforderungen kodiert (Tabelle 2). Der sozioökonomische Status der Eltern wurde siebenstufig klassifiziert (Brauns et al.,
1997). Das allgemeine soziale Funktionsniveau wurde mit der Global Assessment of Function Scale (GAF, American Psychiatric Association, 1993) eingeschätzt.

Zur Untersuchung neuropsychologischer Einbußen kamen Verfahren zur Anwendung, mit denen die verbale Intelligenz sowie die bei Schizophrenie häufigen Defizite erfasst werden können und die in internationalen Studien verbreitet sind (Frangou, 2010; Szöke et al., 2008). Gleichzeitig mussten sie unter den Bedingungen der Regelbetreuung durchführbar sein. Fünf Subtests des HAWIE-R (Tewes, 1994) bzW. des HAWIKIII (Tewes, Schallberger \& Rossmann, 1999) wurden angewendet: Allgemeines Wissen (AW), Rechnerisches Denken (RD), Zahlennachsprechen (ZN), Mosaiktest (MT), Zahlen-Symbol-Test (ZS). Psychomotorische Geschwindigkeit und selektive Aufmerksamkeit wurden mit dem Pfadfindertest (TMT; Reitan, 1956), Wortflüssigkeit mit dem FAS-Test (Benton \& Hamsher, 1976) und das Gedächtnis mit dem Wechsler Gedächtnistest - revidierte Fassung (WMS-R; Härting, Markowitsch, Neufeld, Calabrese \& Deisinger, 2000) geprüft.

Die psychopathologischen Einschätzungen erfolgten durch erfahrene klinische Psychologen der Universitätsklinik für Psychiatrie und Psychotherapie des Kindes und Jugendalters. Die neuropsychologischen Tests wurden von wissenschaftlichen Hilfskräften dieser Klinik durchgeführt, die über die Studie sonst nicht informiert waren. Die Ergebnisse beider Untersuchungen wurden erst nach der Datenerhebung verglichen.

Die Statistische Analyse erfolgte mit der Software SPSS 15. Es wurden Unterschiede zwischen den Gruppen zu beiden Testzeitpunkten und Unterschiede innerhalb jeder Gruppe zwischen den Testzeitpunkten untersucht. Kategorien 
wurden mittels $X^{2}$-Tests, Rang-Skalen mit Kendall $\tau-c$ (dieses Verfahren toleriert leere Zellen) ausgewertet. Unterschiede kontinuierlicher Variablen wurden varianzanalytisch untersucht. Wenn die Varianzen nicht homogen waren, wurde der $\mathrm{t}$-Test verwendet. Veränderungen zwischen T1 und T2 wurden mit einer Varianzanalyse für abhängige Stichproben (Messwiederholungen) geprüft. Bei kontinuierlichen Variablen wurde die Interaktion zwischen Gruppen und Testzeitpunkten mit zweifaktoriellen Varianzanalysen für abhängige Stichproben (Messwiederholungen) geprüft. Da jeweils zwei Variablen gegeneinander getestet wurden war eine Korrektur für multiples Testen nicht erforderlich. Zur Bestimmung von Effektstärken (Power) wurde Hedges' unbiased g (Hedges \& Olkin, 1985) berechnet, um den Vergleich mit anderen Untersuchungen zu ermöglichen. Maße der Effektstärke beziehen sich auf Unterschiede in Bezug zur über beide Testzeitpunkte gemittelten Standardabweichung $(S D=1)$. Ein positiver Wert der Effektstärke bezeichnet eine Veränderung in die erwünschte Richtung. Ein Wert von 0.2 wäre eine geringe, 0.5 eine mittlere und ab 0.8 eine hohe Effektstärke.

\section{Ergebnisse:}

Es zeigten sich keine signifikanten Unterschiede zwischen den Gruppen hinsichtlich Diagnose, Geschlechterverhältnis, Alter oder Medikation (Chlorpromazinäquivalente, Tabelle 1). Verordnet wurden Clozapin (28\%), Olanzapin (23\%), Amisulprid (17 \%), Quetiapin (15\%), Risperidon (8\%), Aripiprazol (4\%), Promethazin (2\%) und Haloperidol (2\%). Zuweisungskriterien von Kliniken und Jugendämtern führten dazu, dass der sozioökonomische Status in der TG geringer war (Kendall- $\tau \mathrm{c}=.528 / p=.005$ ).

Der Einzug in die WG Trialog erfolgte im Mittel 0.94 (Min. 0.43, Max. 1.85) Jahre nach der ersten Aufnahme. In diesem Zeitraum wurden $75 \%$ der Patienten bereits mehrfach (Durchschnitt 2.1) aufgenommen. 62\% dieses Zeitraumes (MW 212, Min. 82, Max. 419 Tage) verbrachten die Patienten in stationärer Behandlung. Daten der Vergleichsgruppe hierzu lagen nicht vor. Die Dauer der unbehandelten Psychose war in der TG signifikant länger als in der VG (Tabelle 1).

Die Psychopathologie zeigte zum Zeitpunkt T1 stärker ausgeprägte positive Symptome in der TG als in der VG. Sie besserten sich in der TG, während sie sich in der VG verschlechterten (Tabelle 3, Interaktion Gruppe $\times$ Zeit: $F(1,22)=$ 24.621, $p<.001$ ). Negative Symptome wurden in beiden Gruppen zu T1 in vergleichbarem Maße eingeschätzt. Nur in der TG zeigten sie eine Tendenz zur Besserung bis T2. Auch die Interaktion Gruppe $\times$ Zeit zeigte einen Trend $(F(1,22)$ $=3.640, p=.07)$. Vergleichbare Besserungen ergaben sich im CGI (Tabelle 3: Interaktion Gruppe $\times$ Zeit: $F(1,22)=$ 8.186, $p=.009$ ).

Die Hälfte der Patienten der TG wurde während des Aufenthaltes für eine Umstellung der Medikation oder wegen einer psychotischen Exazerbation stationär aufgenommen (23 Tage pro Jahr). Sieben Patienten der VG (58\%) wurden im gleichen Zeitraum erneut stationär aufgenommen.

Das neuropsychologische Funktionsniveau (Tabelle 4) zeigte keine Unterschiede oder Veränderungen in den Subtests zur verbalen Intelligenz. Im Bereich der Aufmerksamkeit und der exekutiven Funktionen tendierte die TG zu niedrigeren Leistungen als die VG im ZS zu $\mathrm{T} 1$. Verbesserungen der $\mathrm{TG}$ von $\mathrm{T} 1$ bis $\mathrm{T} 2$ zeigten sich im MT (visuo-motorische Koordination und Flexibilität), ZS (Trend) und TMT-A (psychomotorisches Tempo), TMTB (selektive Aufmerksamkeit, Trend) sowie im FAS (Wortflüssigkeit). In der VG 
Tab. 3: Die Mittelwerte der PANSS Symptom- bzw. CGI Ratings und der Global Assessment of Function Scale (GAF) der Trialog-Gruppe (TG) u. der Vergleichsgruppe (VG) der Untersuchungen zu T1 u. T2 (Standardabweichungen in Klammern)

\begin{tabular}{|c|c|c|c|}
\hline & TG $(\mathbf{N}=12)$ & VG $(N=12)$ & \multirow{4}{*}{  } \\
\hline \multicolumn{3}{|c|}{ Positive Symptome } & \\
\hline T1 & $17.5(5.0)^{1 \mathrm{a}}$ & $12.3(4.9)^{b}$ & \\
\hline T2 & $10.3(2.5)^{2}$ & $14.8(5.9)$ & \\
\hline \multicolumn{4}{|c|}{ Negative Symptome } \\
\hline T1 & $20.8(8.0)^{\mathbf{c}}$ & $23.42(59)$ & c. $\mathrm{T} 1>\mathrm{T} 2: \quad \mathrm{F}_{1,11}=4.279, \mathrm{p}=.063$ \\
\hline T2 & $14.8(5.9)^{3}$ & $24.67(2.1)$ & 3. $\mathrm{TG}<\mathrm{CG}: \mathrm{F}_{1,22}=11.970 \mathrm{p}=.002$ \\
\hline \multicolumn{4}{|c|}{ Clinical Global Impression } \\
\hline T1 & $4.4(0.8)^{4 d}$ & $5.1(0.8)$ & \multirow{2}{*}{$\begin{array}{l}\text { 4. } \mathrm{TG}<\mathrm{CG}: \mathrm{F}_{1,22}=4.241, \quad \mathrm{p}=.051 \\
\text { d. } \mathrm{T} 1>\mathrm{T} 2: \quad \mathrm{F}_{1,11}=42.429, \mathrm{p}=.000 \\
\text { 5. } \mathrm{TG}<\mathrm{CG}: \mathrm{F}_{1,22}=29.607, \mathrm{p}=.000\end{array}$} \\
\hline $\mathbf{T 2}$ & $2.9(0.7)^{5}$ & $4.9(1.1)$ & \\
\hline \multicolumn{4}{|c|}{ Global Assessment of Functioning Scale } \\
\hline T1 & $47.3(11.8)^{\mathbf{a}}$ & $49.6(11.8)$ & \multirow{2}{*}{$\begin{array}{lll}\text { 6. } T G>C G: & F_{1,22}=16.553, & p=.001 \\
\text { a. } T 1<T 2: & F_{1,11}=81.949, & p=.000\end{array}$} \\
\hline $\mathbf{T 2}$ & $68.4(9.5)^{1}$ & $48.3(14.3)$ & \\
\hline
\end{tabular}

Tab. 4: Neuropsychologische Ergebnisse: Mittelwerte (M) Standardabweichungen (SD) von TG u. VG: Testungen zu T1/T2

\begin{tabular}{|c|c|c|c|c|c|c|c|c|c|c|}
\hline Test & $\begin{array}{l}\text { Zeit } \\
\text {-Punkt }\end{array}$ & $\begin{array}{l}\text { Gruppe } \\
\text { TG -- M }\end{array}$ & SD & $\mathrm{N}$ & $\begin{array}{l}\text { Gruppe } \\
\text { VG -- M }\end{array}$ & SD & $\mathrm{N}$ & \multicolumn{3}{|c|}{ statistischer Vergleich } \\
\hline \multirow[t]{2}{*}{ geschätzter IQ } & $\mathrm{T} 1$ & 83.0 & $(11.8)$ & \multirow[t]{2}{*}{12} & 94.7 & $(22.1)$ & \multirow[t]{2}{*}{12} & \multirow{2}{*}{\multicolumn{3}{|c|}{ kein signifikanter Unterschied }} \\
\hline & $\mathrm{T} 2$ & 88.2 & $(17.8)$ & & 92.6 & $(21.5)$ & & & & \\
\hline \multirow{2}{*}{$\begin{array}{l}\text { Zahlennach- } \\
\text { sprechen }\end{array}$} & $\mathrm{T} 1$ & 8.3 & $(2.7)$ & \multirow[t]{2}{*}{12} & 9.5 & $(2.3)$ & \multirow[t]{2}{*}{12} & \multirow{2}{*}{\multicolumn{3}{|c|}{ kein signifikanter Unterschied }} \\
\hline & $\mathrm{T} 2$ & 8.4 & $(1.9)$ & & 9.8 & $(2.7)$ & & & & \\
\hline \multirow[t]{2}{*}{ Mosaik-Test } & $\mathrm{T} 1$ & 8.7 & $(2.6)^{\mathrm{a}}$ & \multirow[t]{2}{*}{12} & 10.3 & $(6.2)$ & \multirow[t]{2}{*}{12} & & & \\
\hline & $\mathrm{T} 2$ & 10.0 & $(3.4)$ & & 11.3 & $(4.1)$ & & a. $\mathrm{T} 1<\mathrm{T} 2$ : & $\mathrm{F}_{1,11}=4.82$ & $\mathrm{p}=.05$ \\
\hline Zahlen- & $\mathrm{T} 1$ & 7.6 & $(2.9)^{1 \mathrm{~b}}$ & \multirow[t]{2}{*}{12} & 10.5 & $(5.1)^{\mathrm{c}}$ & \multirow[t]{2}{*}{12} & 1. $\mathrm{TG}<\mathrm{CG}$ : & $\mathrm{F}_{1,22}=3.02$ & $\mathrm{p}=.096$ \\
\hline Symbol-Test & $\mathrm{T} 2$ & 9.3 & $(3.3)$ & & 8.7 & $(4.6)$ & & $\begin{array}{l}\text { b. } \mathrm{T} 1<\mathrm{T} 2: \\
\text { c. } \mathrm{T} 1>\mathrm{T} 2:\end{array}$ & $\begin{array}{l}\mathrm{F}_{1,11}=3.60 \\
\mathrm{~F}_{1,11}=7.97\end{array}$ & $\begin{array}{l}\mathrm{p}=.084 \\
\mathrm{p}=.017\end{array}$ \\
\hline \multirow{2}{*}{$\begin{array}{r}\text { TMT - A } \\
\text { (Sek) }\end{array}$} & $\mathrm{T} 1$ & 31.3 & $(9.4)^{\mathrm{d}}$ & \multirow[t]{2}{*}{10} & 36.40 & $(13.8)$ & \multirow[t]{2}{*}{10} & \multirow[t]{2}{*}{ d. $\mathrm{T} 1>\mathrm{T} 2$ : } & \multirow[t]{2}{*}{$\mathrm{F}_{1,9}=5.64$} & \multirow[t]{2}{*}{$\mathrm{p}=.03$} \\
\hline & $\mathrm{T} 2$ & 25.0 & $(9.8)$ & & 45.8 & $(47.2)$ & & & & \\
\hline \multirow{2}{*}{$\begin{array}{c}\text { TMT - B } \\
\text { (Sek) }\end{array}$} & $\mathrm{T} 1$ & 84.4 & $(27.4)^{\mathrm{e}}$ & \multirow[t]{2}{*}{12} & 76.4 & $(26.3)^{f}$ & \multirow[t]{2}{*}{10} & \multirow{2}{*}{\multicolumn{2}{|c|}{$\begin{array}{l}\text { e. } \mathrm{T} 1>\mathrm{T} 2 \text { : } \\
\text { f. } \mathrm{T} 1<\mathrm{T} 2 \text { : }\end{array}$}} & $\mathrm{p}=.095$ \\
\hline & $\mathrm{T} 2$ & 76.3 & $(29.5)$ & & 94.7 & $(36.9)$ & & & & $\mathrm{p}=.079$ \\
\hline \multirow[t]{2}{*}{ TMT B-A } & $\mathrm{T} 1$ & 56.1 & $(24.6)$ & \multirow[t]{2}{*}{12} & 44.1 & $(20.3)^{j}$ & \multirow[t]{2}{*}{10} & \multirow[t]{2}{*}{ j. $\quad \mathrm{T} 1<\mathrm{T} 2$ : } & \multirow[t]{2}{*}{$\mathrm{F}_{1,9}=3.81$, } & $\mathrm{p}=.087$ \\
\hline & $\mathrm{T} 2$ & 51.1 & $(25.5)$ & & 64.1 & $(30.0)$ & & & & \\
\hline Wortflüssigkeit & $\mathrm{T} 1$ & 23.5 & $(9.9)^{2}$ & 12 & 21.8 & $(4.9)$ & 10 & 2: T1-T2: & $\mathrm{F}_{1,10}=6.16$ & $\mathrm{p}=.018$ \\
\hline Anzahl Wörter & $\mathrm{T} 2$ & 25.5 & $(5.9)$ & & 22.5 & $(8.5)$ & & & & \\
\hline Visuelles Gedächt & itnis & & & & & & & & & \\
\hline Sofortige & $\mathrm{T} 1$ & 33.3 & $(4.4)^{3}$ & 12 & 36.7 & $(3.4)^{\mathbf{g}}$ & 11 & 3. $\mathrm{TG}<\mathrm{CG}$ : & $\mathrm{F}_{1,21}=4.25$ & $\mathrm{p}=.052$ \\
\hline Reproduktion & $\mathrm{T} 2$ & 34.7 & $(5.1)$ & & 34.5 & $(7.0)$ & & g. T1>T2: & $\mathrm{F}_{1,10}=4.25$ & $\mathrm{p}=.052$ \\
\hline Verzögerte & $\mathrm{T} 1$ & 28.9 & $(7.3)^{h}$ & 12 & 33.6 & $(9.6)$ & 11 & h. $\mathrm{T} 1<\mathrm{T} 2$ : & $\mathrm{F}_{1,11}=4.50$ & $\mathrm{p}=.04$ \\
\hline Reproduktion & $\mathrm{T} 2$ & 33.6 & $(5.6)$ & & 31.7 & $(10.5)$ & & & & \\
\hline Verbales Gedächt & itnis & & & & & & & & & \\
\hline Sofortige & $\mathrm{T} 1$ & 17.3 & $(4.6)^{4}$ & 12 & 24.3 & $(8.9)$ & 12 & 4. $\mathrm{TG}<\mathrm{CG}$ : & $\tau=-2.42$ & $\mathrm{p}=.028$ \\
\hline Reproduktion & $\mathrm{T} 2$ & 21.4 & $(8.3)$ & & 22.8 & (10.9) & & & & \\
\hline Verzögerte & $\mathrm{T} 1$ & 13.3 & $(4.1)^{5 \mathbf{i}}$ & 12 & 19.3 & $(8.4)$ & 12 & 5. $\mathrm{TG}^{8}<\mathrm{CG}$ : & $\tau=-2.23$ & $\mathrm{p}=.04$ \\
\hline Reproduktion & $\mathrm{T} 2$ & 19.3 & $(8.1)$ & & 17.4 & $(9.9)$ & & i. $\mathrm{T} 1<\mathrm{T} 2$ : & $\mathrm{F}_{1,11}=4.759$ & $\mathrm{p}=.035$ \\
\hline
\end{tabular}






Abb. 1: Geschätzte Effektstärken signifikanter Veränderungen der TG, VG und 95\% Vertrauensintervallgrenzen von AOS-Patienten (Hedges' unbiased g, Szöke et al. 2008)

ergaben sich Verschlechterung im ZS, TMT-B (Trend) und TMT B-A (kognitive Flexibilität, Trend). Die Analyse der Interaktion von Gruppe und Zeit war signifikant hinsichtlich einer günstigeren Entwicklung in der TG als in der VG im TMT-B $(F(1,18)=5.420, p=.031)$ und im ZS $(F(1,22)=10.094, p=.004)$.

Sofortige visuelle und verbale Gedächtnisleistung war in der TG zu T1 stärker beeinträchtigt als in der VG. Zu T2 bestand kein Unterschied mehr. Die Leistung der VG verschlechterte sich in der sofortigen visuellen Reproduktion. Die verzögerte visuelle und verbale Reproduktion verbesserte sich in der TG, während sie sich in der VG nicht veränderte. Die Interaktion von Gruppen und Testzeitpunkten war signifikant (visuell: $F(1,21)=8.764 / p=.007$; verbal: $F(1,22)=7.188 / p=.014)$.

Die Effektstärken (Hedges' unbiased g) der Verbesserungen neuropsychologischer Testergebnisse konnten mit Testwiederholungsergebnissen ohne spezielles Training von AOS-Patienten (Szöke et al., 2008) verglichen werden (Abbildung 1). Die für die jeweiligen Tests gefundenen 95
\%-Konfidenzintervalle von Hedges' unbiased $g$ wurden im MT, ZS, TMT-A, in sofortiger und verzögerter verbaler sowie verzögerter visueller Reproduktion überschritten. Das spricht dafür, dass die Wirksamkeit der hier untersuchten Betreuung die durch eine Testwiederholung eintretende Verbesserung durch Übung, durch die erfolgte Behandlung (TAU) und Zufallseffekte bei den genannten Tests mit hoher Wahrscheinlichkeit übersteigt. Die Effektstärken der VG-Ergebnisse lagen mit Ausnahme vom MT in allen Testergebnissen unter diesem Bereich.

Bezüglich des sozialen Funktionsniveaus zeigten die Wohnbedingungen der TG nach Abschluss der Betreuung ein höheres $M a ß$ an Selbstständigkeit. Es wurden häufiger regelmäßige Tätigkeiten ausgeübt als in der VG. Die erreichten Schulabschlüsse waren trotz teilweise niedrigerer neuropsychologischer Fähigkeiten der TG in beiden Gruppen vergleichbar (Tabelle 2). Die GAF-Ratings lagen zu Beginn der Studie auf einem ähnlich niedrigen Niveau in beiden Gruppen. In der TG zeigten sie bis T2 einen signifikanten Anstieg, während sie in der 
VG auf dem Ausgangsniveau blieben (Tabelle 3: Interaktion Gruppe $\times$ Zeit $F(1$, 22) $=17.248, p=.001$ ).

Die Lebensqualität (MANSA) zeigte keine Unterschiede der subjektiven Zufriedenheit mit der Lebenssituation, der Freizeitgestaltung, der finanziellen Situation, der Wohnsituation, der Gesundheit und dem Kontakt zu Freunden. Nur die Zufriedenheit mit den Beziehungen zur Familie stieg tendenziell in der TG $(M(S D)$ T1 < T2: 5.0 (1.7)/6.2 (1.3); $F(1,11)=4.568, p=.058)$. In der VG fand sich keine signifikante Veränderung (T1: 5.3 (1.0), T2: 4.9 (1.8)). Die Interaktion Gruppe $\times$ Zeit war signifikant

\section{Limitationen}

Diese Untersuchung stützt sich auf eine kleine Stichprobe. Eine Verblindung bezüglich der Testzeitpunkte war nicht möglich. Auch eine Randomisierung und Parallelisierung von Interventions- und Vergleichsgruppe war nicht möglich, da aufgrund der niedrigen Prävalenz und aus ethischen Gründen alle vorgestellten Betroffenen um Teilnahme gebeten wurden. Psychopathologische Symptome wurden mit unterschiedlichen Verfahren untersucht (VG: SANS/SAPS, TG: PANSS). Die PANSS wurde in der später begonnen Evaluation verwendet, da sie sich international durchsetzt und ein Vergleich mit anderen Studien, vor allem bei weiteren Verlaufsuntersuchungen, erleichtert wird.

\section{Diskussion}

In dieser Studie wurde der Verlauf von Psychopathologie, Lebensqualität, sozialem Funktionsniveau und Leistung in neuropsychologischen Tests bei je zwölf EOS-Patienten in zweijähriger spezialisierter Betreuung (TG) und unter einem TAU (VG) drei Jahre nach Beginn der Erkrankung verglichen. Die Betreuung verringerte psychopathologische Symptome in höherem Maße als das TAU und besserte das soziale und in einigen Bereichen das neuropsychologische Funktionsniveau mehr als das TAU, auch bei Betroffenen mit prädiktiv ungünstigen Merkmalen. Psychopathologische und neuropsychologische Merkmale der Teilnehmer entsprachen Ergebnissen, wie sie auch von anderen Autoren bei EOSPatienten gefunden wurden (Biswas et al., 2006a; Biswas, Malhotral, Malhotral \& Nitin, 2006b; Mayoral et al., 2008). Die Entwicklung neuropsychologischer Fähigkeiten ohne Intensivbetreuung ist anderen Studien zufolge weniger vorteilhaft (Übersicht bei Frangou, 2010).

Betreuung in der WG Trialog wurde vorwiegend für Patienten gesucht, die kurz nach der ersten Entlassung erneut aufgenommen werden mussten, die aus Familien mit eingeschränkten sozioökonomischen Ressourcen stammten und deren neuropsychologische Fähigkeiten in manchen Bereichen stärker beeinträchtigt waren. Auch die Dauer der unbehandelten Psychose, zu der es vor allem bei einem prognostisch ungünstigen schleichenden Beginn der Erkrankung kommt (Röpcke \& Eggers, 2005), war in der TG länger als in der VG. Eingeschränkte sozioökonomische Ressourcen zeigen eine Verbindung mit einer höheren Inzidenz von Typen schizophrener Störungen mit ungünstigem Verlauf (Gallagher, Jones, McFalls und Pisa, 2005; Straube \& Oades, 1992). Für die TG erfolgte eine Selektion von Patienten mit prädiktiv ungünstigeren Merkmalen. Fünf Bewohner der WG Trialog schlossen die Betreuung aufgrund fehlender Kooperation nicht ab, in drei Fällen stand dies in Verbindung mit Drogenkonsum. Dieses Problem besteht, seit psychosoziale Interventionen angeboten werden (Mayr-Mauhardt, 2005; Straube \& Oades, 1992). Die Berücksichtigung des Stadiums der Verarbeitung der Störung der gesamten Familie verbessert die Effektivität (Glynn, Cohen \& Niv, 2007; Vauth, Bull \& Schneider, 2009) 
und verringerte die Zahl der Abbrüche der Betreuung in der WG Trialog auf einen in den letzten zwei Jahren.

Eine Besserung neuropsychologischer Defizite, wie sie hier gefunden wurde, geht bei AOS nicht nur mit einem Anstieg des sozialen Funktionsniveaus einher, sondern hat sich auch für die Lebensqualität von größerer Bedeutung erwiesen als ein Rückgang psychopathologischer Symptome. Ihre Besserung schafft die Voraussetzung für einen guten Verlauf der Schizophrenie (Hofer et al., 2005; Helldin, Kane, Karilampi, Norlander \& Archer, 2006). Neuropsychologische Störungen stehen in engem Zusammenhang mit negativen Symptomen (Silverstein, Mavrolefteros \& Close, 2002), die durch eine erfolgreiche neuropsychologische Rehabilitation ebenfalls gebessert werden können (Villalta-Gil et al., 2006).

Die Zahl stationärer Wiederaufnahmen ging im Vergleich zu dem Jahr vor dem Einzug in der WG Trialog von $75 \%$ auf $25 \%$ pro Jahr zurück. Röpcke und Eggers (2005) fanden in einer früheren Stichprobe von 39 EOS-Patienten aus der gleichen Region eine Wiederaufnahmerate von $47 \%$ im ersten Jahr nach der Entlassung, was die Neigung zur Auswahl schwerer gestörter Patienten für die TG bekräftigt. Die geringere Wiederaufnahmerate unter Intensivbetreuung kann größerer Stabilität des psychischen Zustandes der Bewohner durch höhere Kontinuität und Vielfältigkeit der Behandlung, rascher Krisenintervention und Vermeidung von Überforderung zugeschrieben werden. In leichteren Krisen konnte die Betreuung vielleicht auch eine klinische Behandlung ersetzen. Die Betreuung in familienzentrierten Wohngruppen führte auch bei AOSPatienten zu einer verringerten Wiederaufnahmerate und verbesserte die psychopathologische Symptomatik (MayrMauhart, 2005). Im Gegensatz dazu konnten in einer Rehabilitationseinrichtung weit entfernt von den Familien erzielte Fortschritte bei EOS-Patienten bei Denkstörungen, affektiven Störungen, positiven und negativen Symptomen nicht aufrechterhalten werden (Fleischhaker et al., 2005; Heinemann, 2005; Martin, 1991).

Die TG erzielte mehr Autonomie in ihrer Lebensgestaltung als die Vergleichsgruppe (Tabelle 2) und als diejenigen, die in weiter Entfernung von ihren Familien betreut wurden (Heinemann, 2005). In der Vergleichsgruppe lebten zu T2 25\% in Wohnheimen, $58 \%$ bei ihren Eltern. Mindestens die Hälfte dieser Eltern konnte ihre Kinder im häuslichen Umfeld nicht ausreichend unterstützen, wodurch sich ihr Zustand verschlechterte. In anderen Studien (Heinemann, 2005) lebten 57\% in Wohnheimen. Im Gegensatz dazu zeigte die Verlaufsstudie einer familienzentrierten Wohngruppe, dass ein Jahr nach Auszug $72 \%$ der früheren Bewohner mit AOS in ihrer eigenen Wohnung lebten (Mayr-Mauhart, 2005).

Die Hälfte der Trialog-Bewohner ging einer regelmäßigen Tätigkeit nach, die sie für eine reguläre Berufstätigkeit vorbereitete (Schule, berufsvorbereitende Maßnahmen, Freiwilliges Soziales Jahr). Zu diesem Zeitpunkt bestand kein Unterschied $\mathrm{zu}$ den $42 \%$ der Vergleichsgruppe, die auf gleichem Niveau tätig waren, aber nur 19\% der oben zitierten Studie (Heinemann, 2005) erreichten acht Jahre nach der ersten Aufnahme dieses Niveau. Nur 7\% dieser Studie erreichten eine reguläre Berufstätigkeit. Dieser Anteil ist niedriger als das Beschäftigungsniveau von $20 \%$, das in der früheren Verlaufsstudie von EOS-Patienten über 15 Jahre erreicht wurde (Röpcke \& Eggers, 2005). Hier zeigt sich, dass es sich auch bei der von Heinemann (2005) untersuchten Stich- 
probe um eine Auswahl schwerer Betroffener handelte, aber auch, dass der Verlauf ihrer Störung langfristig nicht gebessert werden konnte. Früheren Verlaufsstudien zufolge blieben etwa die Hälfte der Patienten mit der Diagnose EOS sozial behindert mit GAF-Werten von 50 oder weniger (Röpcke \& Eggers, 2005). Dies wurde bekräftigt durch den Anteil von ebenfalls $50 \%$ von Patienten der Vergleichsgruppe zu T2 und 56\% der Stichprobe von Heinemann (2005) bei der Katamnese nach acht Jahren, die einen GAF-Wert von unter 50 hatten. Die Teilnehmer der TG hatten alle eine GAF von über 50 .

Veränderungen der subjektiven Lebensqualität wurden nur für die Zufriedenheit mit den Beziehungen zur Familie in der TG gefunden. Der enge Kontakt und die Ermutigung zur Beteiligung und Mitverantwortung der Familien, wie sie in der WG Trialog gepflegt werden, führten meist zu einer konstruktiven Zusammenarbeit.

Wie bei AOS-Patienten scheint intensive Familienarbeit auch bei EOSPatienten den Verlauf verbessern zu können. Zum Teil kann dies einer höheren Kontinuität der Medikamenteneinnahme in der TG zugeschrieben werden. Unterbrechungen der Einnahme kamen auch bei Teilnehmern der TG vor, fielen jedoch schneller auf als unter einem TAU. Es erfolgte keine ständige Überwachung. Die eigenverantwortliche Einnahme wurde in kleinen Schritten im individuellen Tempo entwickelt. Auch hierzu diente die intensive und langfristige Auseinandersetzung mit (Neben-) Wirkung, Symptomatik und Belastungsgrenzen mit Betroffenen und Angehörigen.

Die beobachtete Verschlechterung des Zustandes der Teilnehmer der VG in einigen Bereichen ging maßgeblich auf vier Patienten mit schlechtem Verlauf zurück. Bei ihnen waren Versuche, eine regelmäßige Tätigkeit aufzunehmen, vermutlich aufgrund von Überforderung bei nicht ausreichender Spezialisierung der Angebote, misslungen.

Spezialisierte, familienzentrierte, befristete und gemeindenahe Betreuung scheint auch für EOS-Patienten wirksamer als bestehende Angebote zu sein, auch bei prognostisch ungünstigen Merkmalen. Sie ist in einer Jugendhilfeeinrichtung der Regelversorgung durchführbar. Weitere Untersuchungen sollen klären, ob und unter welchen Voraussetzungen diese Fortschritte aufrechterhalten und weitergehende Aufenthalte in stationären Einrichtungen vermieden werden können.

Literatur:

American Psychiatric Association. (1993). Diagnostic and statistical manual of mental disorders (4th ed.). Washington, DC: American Psychiatric Association.

Andreasen, N. C. (1983). Scale for the Assessment of Negative Symptoms (SANS). lowa City: University of lowa.

Andreasen, N.C. (1984). Scale for the Assessment of Positive Symptoms (SAPS). lowa City: University of lowa.

Benedetti, G. (1998). Psychotherapie als existentielle Herausforderung. Göttingen: Vandenhoeck \& Ruprecht.

Benedetti, G. (2004). Mirror image experiences in psychosis therapy. InD. Bürgin \& H. Meng (Eds.), Childhood and adolescent psychosis. Basel: Karger.

Benton, A. L. \& Hamsher, K. (1976). Multilingual aphasia examination. lowa City: University of lowa.

Biswas, P., Malhotral, S., Malhotral, A. \& Nitin, G. (2006a). Comparative study of neuropsychological correlates in schizophrenia with onset in childhood, adolescence and adulthood. European Child and Adolescent Psychiatry, 15, 360-366.

Biswas, P., Malhotral, S., Malhotral, A. \& Nitin, G. (2006b). Comparative study of clinical correlates in schizophrenia with onset in childhood, adolescence and 
adulthood. Journal of Indian Association for Child and Adolescent Mental Health, 2(1), 18-30.

Brauns, H. D., Haun, D. \& Steinemann, S. (1997). Die Konstruktion eines international vergleichbaren Klassenschemas (EGP). Erwerbsstatische Besonderheiten am Beispiel von Labour Force Surveys der Bundesrepublik Deutschland, Frankreichs, Großbritanniens und Ungarns. Mannheimer Zentrum für Europäische Sozialforschung, Working Paper Series 1/22.

Ciompi, L., Hoffmann, H. \& Broccard, M. (Hrsg.). (2001). Wie wirkt Soteria? Bern: Huber.

Eggers, C. (2004). Grundzüge der Therapie. In C. Eggers, J.M. Fegert \& F. Resch (Hrsg.), Psychiatrie und Psychotherapie des Kindes- und Jugendalters (S. 145203). Heidelberg: Springer-Verlag.

Eggers, C., Bunk, D., Volberg, G. \& Röpcke, B. (1999). The Essen study of childhood onset schizophrenia: Selected results. European Child and Adolescent Psychiatry, 8(Suppl. 1), 21-28.

Falkai, P. (2008). Diagnose, Ätiologie und Neuropathophysiologie der Schizophrenie. In T. Kircher \& S. Gauggel (Hrsg.), Neuropsychologie der Schizophrenie (S. 36-43). Heidelberg: Springer-Verlag.

Fleischhaker, C., Schulz, E., Tepper, K., Martin, M., Hennighausen, K. \& Remschmidt, H. (2005). Long-term course of adolescent schizophrenia. Schizophrenia Bulletin 31, 769-780.

Frangou, S. (2010). Cognitive function in early onset schizophrenia: A selective review. Human Neuroscience, 3, 1-6.

Gallagher, B. J. 3rd, Jones, B. J., McFalls, J. A. Jr. \& Pisa, A.M. (2005). Social class and type of schizophrenia. European Psychiatry 21, 233-237.

Glynn, S., Cohen, A. N. \& Niv, N. (2007). New challenges in family interventions for schizophrenia. Expert Review of Neurotherapeutics, 7, 33-43.
Härting, C., Markowitsch, H. J., Neufeld, H., Calabrese, P. \& Deisinger, K. (2000). Wechsler Gedächtnis Test - revidierte Fassung. Göttingen: Hogrefe.

Hedges, L.V. \& Olkin, I. (1985). Statistical methods for metaanalysis. Orlando: Academic Press.

Heinemann, G. (2005). Die Bedeutung prämorbider und psychopathologischer Parameter für den Verlauf schizophrener Psychosen in der Adoleszenz zu unterschiedlichen Zeitpunkten. Inaugural-Dissertation, Philipps-Universität Marburg.

Helldin, L., Kane, J. M., Karilampi, U., Norlander T. \& Archer, T. (2006). Remission and cognitive ability in a cohort of patients with schizophrenia. Journal of Psychiatry Research, 40, 738-745.

Hemmerle, M.J. (2008). Vergleich der Wirksamkeit einer zweijährigen Intensivbetreuung von Schizophrenen mit frühem Erkrankungsbeginn mit einem Treatment-as-usual. Inaugural Dissertation, Universität DuisburgEssen. http://duepublico.uni-duisburgessen.de/servlets/DerivateServlet/Deri vate-1587/Dissertation_Hemmerle.pdf

Hofer, A., Baumgartner, S., Bodner, T., Edlinger, M., Hummer, M., Kemmler, G., Fleischhaker,W. W. (2005). Patient outcomes in schizophrenia II: The impact of cognition. European Psychiatry, 20, 395-402.

Katschnig, H., Konieczna, H., Michelbach, H. \& Sint, P.P. (1989). Intimität und Distanz - ein familienorientiertes Wohnheim für schizophrene Patienten. In H. Katschnig, (Hrsg.). Die andere Seite der Schizophrenie - Patienten zu Hause (S. 229-242). München: PsychologieVerlags-Union.

Kay, S. R. (1987). The Positive and Negative Syndromes Scale (PANSS) for schizophrenia: Development and standardization. Schizophrenia Bulletin 13, 261-267. 
Kircher, T. \& Gauggel, S. (Hrsg.). (2008). Neuropsychologie der Schizophrenie. Heidelberg: Springer-Verlag.

Martin, M. (1991). Verlauf der Schizophrenie im Jugendalter unter Rehabilitationsbedingungen. Stuttgart: Enke.

Mayoral, M., Zabala, A., Robles, O., Bombín, I., Andrés, P., Parellada, M., Arango C. (2008). Neuropsychological functioning in adolescents with first episode psychosis: A two-year followup study. European Psychiatry 23, 375383.

Mayr-Mauhart, M. (2005). Die Langzeiteffekte der Teilnahme an einem familienorientierten Wohnheimprojekt für Familien mit einem an Schizophrenie erkrankten Mitglied. InauguralDissertation der Medizinischen Fakultät, Universität Wien.

McFarlane, W. R., Dushay, R. A., Stastny, P., Deakins, S. M. \& Link, B. (1996). A comparison of two levels of familyaided assertive community treatment. Psychiatric Services 47, 744-750.

Norman, R. M., Malla, A. K., Cortese, L. \& Diaz, F. (1996). A study of the interrelationship between and comparative interrater reliability of the SAPS, SANS and PANSS. Schizophrenia Research, 19, 73-85.

Oades, R. D., Wild-Wall, N., Juran, S. A., Sachsse, J., Oknina, L. B. \& Röpcke, B. (2006). Auditory change detection in schizophrenia: Sources of activity, related neuropsychological function and symptoms in patients with a first episode in adolescence, and patients 14 years after an adolescent illness-onset. BioMed Central Psychiatry, 6, 7.

Oknina, L. B., Wild-Wall, N., Oades, R. D., Juran, S. A., Röpcke, B., Pfueller, U., Weisbrod, M., Chan, E., Chen, E. Y. H. (2005). Frontal and temporal sources of mismatch negativity in healthy controls, patients at onset of schizophrenia in adolescence and others at 15 years after onset. Schizophrenia Research, 76, 25-41.

Priebe, S., Huxley, P., Knight, S. \& Evans, S. (1999). Application of the Manchester Short Assessment of Quality of Life (MANSA). International Journal of Social Psychiatry, 45, 7-12.

Reitan, R.M. (1956). Trail Making Test: Manual for administration, scoring and interpretation. Indianapolis: Indiana University Press.

Röpcke, B. \& Eggers, C. (2005). Early-onset schizophrenia - a 15-year follow-up. European Child and Adolescent Psychiatry 14, 341-350.

Silverstein, M.L., Mavrolefteros, G. \& Close, D. (2002). Premorbid adjustment and neuropsychological performance in schizophrenia. Schizophrenia Bulletin, 28, 157-165.

Straube, E. \& Oades, R. D. (1992). Schizophrenia: Empirical research and findings. New York: Academic Press.

Szöke, A., Trandafir, A., Dupont, M.E., Méary, A., Schürhoff, F. \& Leboyer, M. (2008). Longitudinal studies of cognition in schizophrenia: Metaanalysis. British Journal of Psychiatry, 192, 248-257.

Tewes, U. (1994). Hamburg-WechslerIntelligenztest für Erwachsene, Revision 1991. Bern: Huber.

Tewes, U., Schallberger, P. \& Rossmann, U. (Hrsg.). (1999). Hamburg-WechslerIntelligenztest für Kinder III (HAWIK III). Bern: Huber.

Vauth, R., Bull, N. \& Schneider, G. (2009). Emotions- und stigmafokussierte Angehörigenarbeit bei psychotischen Störungen: Ein Behandlungsprogramm. Göttingen: Hogrefe.

Villalta-Gil, V., Vilaplana, M., Ochoa, S., Haro, J. M., Dolz, M., Usall, J. \& the NEDENA Group. (2006). Neurocognitive performance and negative symptoms: Are they equal in explaining disability in schizophrenia outpatients? Schizophrenia Research, 87, 246-253. 
White T., Ho B.C., Ward J., O'Leary D. \& Andreasen N.C. (2006). Neuropsychological performance in first-episode adolescents with schizophrenia: A comparison with first-episode adults and adolescent control subjects.

Biological Psychiatry, 60, 463-471. 